\title{
Role of lymphangiogenesis in epithelial ovarian cancer
}

\author{
SS Sundar', H Zhang', P Brown², S Manek², C Han ${ }^{3}$, K Kaur $^{3}$, MFL Charnock ${ }^{4}$, D Jackson ${ }^{5}$ and TS Ganesan*, I,6 \\ 'Ovarian Cancer Group, Cancer Research UK Molecular Oncology Laboratories, Weatherall Institute of Molecular Medicine, John Radcliffe Hospital, \\ Oxford OX3 9DS, UK: ${ }^{2}$ Department of Cellular Pathology, John Radcliffe Hospital, Oxford OX3 9DS, UK; ${ }^{3}$ Cancer Research UK, Churchill Hospital, \\ Headington, Oxford OX3 7LF, UK; ${ }^{4}$ Department of Obstetrics and Gynaecology, John Radcliffe Hospital, Headington, Oxford OX3 9DS, UK; \\ ${ }^{5}$ MRC Human Immunology Unit, Weatherall Institute of Molecular Medicine, John Radcliffe Hospital, Headington, Oxford OX3 9DS, UK
}

We investigated the significance of lymphatic count, vascular count and angiogenic growth factors using immunohistochemistry in 108 tumour specimens of epithelial ovarian cancer with antibodies to lymphatic vessel endothelial hyaluronan receptor (LYVE-I), platelet endothelial cell adhesion molecule CD3I, vascular endothelial growth factor (VEGF) and thymidine phosphorylase (TP) in epithelial ovarian cancer to understand the pathogenesis of metastasis in ovarian cancer. The effect of prognostic variables on progression-free and overall survival was assessed. On multivariate analysis, bulky residual disease after surgery was the best prognostic indicator $(P<0.00 \mathrm{I})$ for progression-free and overall survival $(P<0.00 \mathrm{I})$. Lymphatic count was statistically significant as a prognostic factor for progression-free $(P=0.05)$ and overall survival $(P=0.04)$. However, lymphatic count did not impact on survival curves. No correlation was found between lymphatic count and age, histological subtype, FIGO stage or residual disease. Vascular count, VEGF or TP expressions were not significant in either analysis. Lymphatic spread may be significant in aiding metastases in ovarian cancer but requires other biological factors to act in conjunction, as it does not have clearcut prognostic significance. Dissemination of ovarian cancer does not occur primarily through vascular or lymphatic routes but may occur through direct intraperitoneal spread of disease. British Journal of Cancer (2006) 94, I650- |657. doi:I0.1038/sj.bjc.6603|44 www.bjcancer.com

Published online 9 May 2006

(c) 2006 Cancer Research UK

Keywords: lymphatic density; vascular density; ovarian carcinoma; angiogenesis; lymphangiogenesis

Epithelial ovarian cancer has an annual incidence of 5000 women in the United Kingdom and is the most important cause of gynaecological cancer-related mortality in the Western world. Owing to the paucity of symptoms and their insidious onset, most women present with advanced disease and 5-year survival rates are approximately 30\% (Agarwal and Kaye, 2003). Management of ovarian cancer in most patients involves surgery to achieve surgical cytoreduction followed by chemotherapy as surgical cytoreduction highly correlates with better patient survival (Cannistra, 2004). Residual disease of more than $2 \mathrm{~cm}$ has traditionally been associated with worse outcome. Sixty to seventy per cent of patients initially respond to platinum-based chemotherapy and approximately $40-50 \%$ achieve a complete clinical remission. However, even in this latter group at least half of the patients experience a recurrence within 4 years. Treatment following relapse after initial chemotherapy is palliative in intent.

It has long been known that some cancers metastasise through lymphatics to the regional lymphnodes before widespread dissemination. This progression from locally confined disease to

* Correspondence: Professor TS Ganesan,

E-mail: tsganesan@aims.amrita.edu

The authors confirm this work to be original and that no parts of this work have been previously published.

${ }^{6}$ Current address: Cancer Institute and Institute of Molecular Medicine, Amrita Institute of Medical Sciences, Elamakkara P.O. Kochi, Kerala, India, 682026, E-mail: tsganesan@aims.amrita.edu

Received 3I October 2005; revised 3 April 2006; accepted 5 April 2006; published online 9 May 2006 lymph node spread confers a worse prognosis for the patient. Lymphangiogenesis, that is, the formation of new lymphatic vessels may play a role in this (Skobe et al, 2001; Stacker et al, 2001; Beasley et al, 2002). Animal models suggest that lymphangiogenesis occurs in malignancy and that inhibiting this process can halt the spread to lymphatics (Makinen et al, 2001; Mandriota et al, 2001; Shimizu et al, 2004). However, tumour cell invasion of pre-existing lymphatics at the tumour margin can also occur (Jackson et al, 2001; Pepper, 2001). Proliferation of lymph vessels is regulated by members of the vascular endothelial growth factor (VEGF) family and their receptors (Oh et al, 1997; Shimizu et al, 2004). VEGF-C and VEGF-D have been identified as stimulators of lymphatic endothelial proliferation acting on VEGF receptor-3, which functions as a specific receptor in adult tissues (Kaipainen et al, 1995). Overexpression of VEGF C and VEGF D in orthotopically transplanted breast carcinoma cells in mice or in VEGF C transgenic mice has been shown to promote tumour lymphangiogenesis and subsequent lymph node metastasis (Pepper, 2001; Skobe et al, 2001; Stacker et al, 2001).

Understanding the role of lymphatics in human cancers has been improved by the recent identification of a number of novel lymphatic receptors - lymphatic vessel endothelial hyaluronan receptor (LYVE-1), Prox 1, podoplanin, $\beta$-chemokine receptor D6, macrophage mannose receptor, desmoplakin that can discriminate lymphatic vessels from blood vessels (Knudson and Knudson, 1993; Breiteneder-Geleff et al, 1999; Wigle et al, 2002; Scavelli et al, 2004). LYVE-1, one of the best studied lymphatic markers is a lymph specific receptor for Hyaluronan (HA) (Banerji et al, 1999) an abundant extracellular matrix glycosylaminoglycan which 
facilitates cell migration in wound healing, tumour metastasis and inflammation by forming a pericellular matrix surrounding fibroblasts and epithelial cells, reducing the level of intercellular adhesion (Knudson and Knudson, 1993; Knudson et al, 1993). LYVE-1 sequesters HA on lymph vessel endothelium, colocalises with HA on the luminal surface of lymphatic vessels and binds both soluble and immobilised HA exclusively (Prevo et al, 2001). LYVE-1 staining of lymphatics is demonstrated in virtually every tissue where these structures could be distinguished and includes vessels draining gastrointestinal tract, skin, lymph nodes, breast and salivary gland and is distinct from blood vessel staining (Banerji et al, 1999). However, LYVE-1 is also expressed in normal liver blood sinusoids in mice and humans (Mouta Carreira et al, 2001).

There is substantial crosstalk between angiogenesis and lymphangiogenesis in tumour progression as evidenced by the involvement of lymphangiogenic factors VEGF-C, D and their receptor VEGFR3 in angiogenesis and the role played by angiogenic factors VEGF-A and Angiopoetin 2 in lymphangiogenesis respectively (Scavelli et al, 2004). Vascular endothelial growth factor is an endothelial cell-specific growth factor and the principal regulator of angiogenesis under normal and pathological conditions in most organs (Ferrara and Alitalo, 1999). Bevacizumab, a VEGF-neutralising antibody has been shown to improve survival in advanced colorectal cancer (Kabbinavar et al, 2005). Thymidine phosphorylase (TP) is often induced in the tumour microenvironment by physiological and chemical stress where it protects cells from apoptosis and helps cell survival by stimulating nucleoside metabolism and angiogenesis (Toi et al, 2005). The degree of angiogenesis of a tumour as measured by vascular density has emerged as a powerful candidate for prognosis and as a predictive tool in breast cancer and melanomas (Bamberger and Perrett, 2002; Uzzan et al, 2004).

This study investigates the significance of tumour lymphatic count and vascular count as well as angiogenic factors VEGF and TP in epithelial ovarian malignancy as prognostic variables to understand the pathogenesis of metastasis in ovarian cancer.

\section{MATERIALS AND METHODS}

\section{Patient data}

The clinical data for this study was collected from unselected patients diagnosed with epithelial ovarian carcinoma between 1990 and 1998 who underwent surgery and treatment at the Oxford Radcliffe Hospitals NHS trust (Oxford, UK). Patients with a histological diagnosis of ovarian carcinoma (borderline tumours were excluded) where follow-up data were known and with sufficient tissue blocks for immunohistochemistry were included. The patients were initially evaluated by clinical, ultrasound examination, computed axial tomography and serum tumour markers. All patients were staged according to FIGO classification (International Federation of Gynaecology and Obstetrics).

Treatment consisted of surgery followed by chemotherapy except in selected patients with Stage 1 disease. Patients who underwent either bilateral salpingoophorectomy with total abdominal hysterectomy and omentectomy or bilateral salpingoophorectomy (where a hysterectomy had been performed previously) were classified as having radical surgery whereas the rest were classified as having debulking surgery to reduce tumour bulk. Residual disease left in situ despite surgery was classified as either microscopic, small volume residual disease $<2 \mathrm{~cm}$ or bulky residual disease $>2 \mathrm{~cm}$ and information was obtained from the operation summary. The majority of patients who received chemotherapy had platinum-based regimens as first-line treatment. Clinical response data for patients who received chemotherapy was assessed as follows - complete remission was defined as the disappearance of all parameters of the disease; partial remission was defined as a $50 \%$ reduction in the size of tumour mass as defined radiologically and progressive disease was defined as a $25 \%$ increase of any tumour mass or the development of a new lesion. Overall and progression-free survival were classified as outcome measures. Overall survival was defined as the period from diagnosis until the time of death from any cause or, in patients who were alive, until 1 June 2004. Progression-free survival was calculated as the time period between diagnosis and relapse of disease. Follow-up data were compiled until 1 June 2004.

Blocks from 93 patients were analysed for determination of lymphatic count and 88 patients for vascular count and angiogenic growth factors with some overlap between the two sets, that is, 74 patients had all parameters assessed. Clinicopathological data are presented from a total of 108 patients. Funding organisations approved but did not influence the conduct of the study. Formal consent was obtained to conduct the study from the hospital research and ethics committee.

\section{Immunohistochemistry}

Representative blocks of ovarian cancer with no normal tissue and no large necrotic areas were selected. Three micrometre sections of formalin-fixed, paraffin-embedded tissue were cut onto glass slides and dried. Sections were dewaxed in xylene, rehydrated through alcohol baths and rinsed in water.

\section{Staining for lymphatic vessel endothelial hyaluronan receptor}

For LYVE-1 staining, slides were then pressure cooked for $2 \mathrm{~min}$ at full pressure in $0.1 \mathrm{~m}$ citrate buffer $(\mathrm{pH}$ 6.0) and rinsed in tap water. The primary antibody (rabbit polyclonal anti-human LYVE-1) was incubated for $60 \mathrm{~min}$ at a 1/400 dilution, followed by $60 \mathrm{~min}$ incubation with the envision polymer (Banerji et al, 1999). The slides were then stained on a Dako Techmate $500^{\mathrm{TM}}$ using Dako EnVision ${ }^{\mathrm{TM}}$ Detection kit Peroxidase/DAB, rabbit/mouse (Dako UK Ltd, Cambridgeshire, UK CB74E1 cat \# K5007). Paraffin sections of normal human small intestine were used as a positive control; slides incubated without primary antibody were used as a negative control.

\section{Immunostaining for platelet endothelial cell adhesion molecule}

The JC70 monoclonal antibody, recognizing the pan-endothelial antigen (platelet cell adhesion molecule, PECAM-1) was used as described previously (Horak et al, 1992). Epitope retrieval was performed by heat section in microwave for $30 \mathrm{~min}$ in trisethylenediaminetetraacetate (EDTA) buffer, and blocked in normal human serum for 30 minutes. Slides were incubated with primary antibody JC70 (Dako, mouse monoclonal, Anti-Human CD31, $1: 50$ ) for $1 \mathrm{~h}$ at room temperature. Horse anti-Mouse IgG at $1: 100$ for $30 \mathrm{~min}$ at room temperature was used as secondary antibody and the slides were developed using diluted horseradish peroxidase (HRP) -Streptavidin. A tissue section of breast carcinoma previously known to demonstrate strong staining was used as positive control; slides incubated without primary antibody were used as a negative control.

\section{Immunostaining for vascular endothelial growth factor and thymidine phosphorylase}

Antigen retrieval was performed using pressure-cooking for $3 \mathrm{~min}$ in Tris-EDTA ( $\mathrm{pH}$ 9) for VEGF, endogenous peroxidase was then quenched with Dako peroxidase block solution for $5 \mathrm{~min}$. The primary antibody (murine monoclonal antibody VG76e/d9) was applied, rinsed in phosphate-buffered saline and then developed 
using the HRP envision system (Dako), as described previously (Turley et al, 1998). Sections were counterstained with haematoxylin and mounted.

For TP immunostaining, no antigen retrieval was performed and was as described previously (Fox et al, 1995). The primary antibody (mouse monoclonal antibody PGF 44-C) was applied as undiluted supernatant for $30 \mathrm{~min}$ at room temperature. After washing in tris-buffered saline, sections were treated with rabbit anti-mouse antibody (Dako) diluted 1 in 50 for $30 \mathrm{~min}$ and then mouse APAAP complex, 1 in 1 , for $30 \mathrm{~min}$. The colour was developed after $15 \mathrm{~min}$ incubation with new fuschsin solution. For VEGF, a section of breast carcinoma previously known to demonstrate strong staining was used as a positive control, for TP tumour-associated macrophages were used as an internal positive control, slides incubated without primary antibody were used as a negative control.

\section{Quantitative analysis of lymphatic vessel, blood vessel density and angiogenic growth factors}

Only vessels with typical irregular morphology and a lumen that stained with LYVE-1 antibody were considered lymphatic vessels as some background staining of macrophages and tumour was noted in some sections. The analysis of lymphatic vessel density (LVD) was not restricted to vessels of any specific diameter as per previous papers (Beasley et al, 2002; Williams et al, 2003). For the microvessel count, small clusters of endothelial cells, with or without a lumen, were considered as individual vessels; singlestained endothelial cells were excluded. Microvessel density (MVD) and LVD were determined in tumour vessel 'hotspots'. The slides were scanned at low magnification (at $\times 10$ and $\times 40$ for lymphatic vessel and microvessel count respectively) and areas of highest lymphatic vessel or MVD were identified as 'hotspots'. Each hotspot was then viewed at high magnification using a $0.25 \mathrm{~mm}^{2}$ microscope ocular grid (at $\times 100$ and $\times 400$ for lymphatic and microvessel, respectively) and the number of vessels counted in five high-power fields per hotspot. This approach has been validated in published literature (Fox and Harris, 2004). The mean of the vessels in three hotspots per slide was recorded. Highest vessel density was also measured. As advanced ovarian cancer penetrates the ovarian capsule, for the purposes of this moral lymphatics (Vermeulen et al, 2002).

Staining for vascular endothelial growth factor was seen in epithelial, macrophage, stromal and vascular tissue and for TP in epithelial and stromal tissue. Expression was assessed by grading each section on staining intensity with subjective scores ranging from 0 to 3, 0 - negative, 1 - weak, 2- moderate and 3 - strong expression as per previous published studies (Van der Auwera et al, 2004). For VEGF, a cumulative score from the four sites of staining was calculated and stratified for analysis $(0-3,4-7,8-12)$. The counts were assessed by an operator without prior knowledge of investigators agreed the count on most of the cases. Cases in disagreement were jointly reviewed under a multiheaded microscope and the consensus count was used for analyses.

\section{Statistics}

The Cox proportional hazards model in the Stata statistical program was used to evaluate the putative prognostic indicators considered (age, stage, residual disease, histological type, grade, LVD and vascular density) and to assess any impact on the outcome measures overall and progression-free survival (Cox and Oakes, 1984). Univariate analysis is presented for all these factors. Factors that were statistically significant on univariate analysis were entered into a model to evaluate the prognostic impact on outcome. For multivariate analysis, individual models with either study no difference was made between intratumoral and peritupatient data and verified by an independent observer. The two

stage or residual disease were computed as both these variables are closely linked, that is, patients with advanced stage disease are more likely to have bulky residual disease after surgery. We also present survival curves using the method of Kaplan and Meier (Kaplan and Meier, 1958).

\section{RESULTS}

\section{Patients}

Clinicopathological data are presented for 108 patients (Table 1). The median overall and progression-free survival was 28.44 and 21 months, respectively. Follow-up information was obtained from

Table I Clinicopathological characteristics of the patients

\begin{tabular}{|c|c|}
\hline Characteristic & All patients \\
\hline Total no. & 108 \\
\hline Age & $25-88$ \\
\hline Median age (years) & 63 \\
\hline \multicolumn{2}{|l|}{ Histological type } \\
\hline Serous & 35 \\
\hline Endometrioid & 34 \\
\hline Clear cell & 16 \\
\hline Mucinous & 12 \\
\hline$M_{M M T^{\mathrm{a}}}$ & 4 \\
\hline Mixed epithelial & 1 \\
\hline Undifferentiated & I \\
\hline Primary peritoneal & 3 \\
\hline Transitional cell carcinoma & I \\
\hline Not known & 1 \\
\hline \multicolumn{2}{|l|}{ Differentiation } \\
\hline Poor & 29 \\
\hline Moderate-poor & 7 \\
\hline Moderate & 49 \\
\hline Well & 20 \\
\hline Not known & 3 \\
\hline \multicolumn{2}{|l|}{ FIGO Stage } \\
\hline I & 32 \\
\hline$\|$ & 13 \\
\hline III & 49 \\
\hline IV & 14 \\
\hline \multicolumn{2}{|l|}{ Surgery } \\
\hline Radical & 59 \\
\hline Debulking & 46 \\
\hline Not known & 3 \\
\hline Residual disease & \\
\hline Microscopic & 53 \\
\hline$\leqslant 2 \mathrm{~cm}$ & 26 \\
\hline$>2 \mathrm{~cm}$ & 25 \\
\hline Not known & 4 \\
\hline \multicolumn{2}{|l|}{ Chemo-response } \\
\hline Complete response & 55 \\
\hline Partial response & 10 \\
\hline Progressive disease & 14 \\
\hline Early death & 13 \\
\hline Not evaluable & 8 \\
\hline Not known & 8 \\
\hline \multicolumn{2}{|l|}{ Chemotherapy } \\
\hline Platinum & 61 \\
\hline Non-platinum & 14 \\
\hline No treatment & 33 \\
\hline
\end{tabular}

British Journal of Cancer (2006) 94(I I), 1650 - 1657 
1990 until June 2004 giving a median follow-up of 28.5 months and a range of $0.49-165.29$ months. A representative mix of histologies and differentiation was noted in our data set. There was a roughly equal distribution of early (Stage I and II) and advanced stage (Stage III and IV) cancers, which is representative of practice at Oxford. After initial surgery, $49 \%$ had microscopic disease, $24 \%$ had residual disease $\leqslant 2 \mathrm{~cm}$ and $23 \%$ had residual disease $>2 \mathrm{~cm}$. Fifty-six per cent of patients received platinum-based chemotherapy, $14 \%$ received nonplatinum-based chemotherapy and $30 \%$ did not receive chemotherapy. Twenty-three of the 108 patients were alive at the time of analysis.

\section{Evaluation of staining}

All but one tumour specimen stained with LYVE-1 antibody. All tumours stained with antibody to CD31 but VEGF and TP staining was more variable (see Supplementary data Table 1 online). Immunostaining with the antibody to the LYVE-1 HA receptor identified irregularly shaped, thin walled lymphatics in the capsular and intratumoural regions of the tumour with capsular lymphatics being more frequent than intratumoural lymphatics. The lymphatics in a section of normal ovary (Figure 1A and B) and tumour stained LYVE-1 antibody (Figure 1C-E). These were distinguished from adjacent blood vessels, which were surrounded by smooth muscle and were regular in shape. Median lymphatic count was 18 (range $0-57$ ). Roughly $52 \%$ of tumours had lymphatic counts below the median, high lymphatic counts (>30) were noted in $16 \%$ of serous, $21 \%$ of endometroid, $7 \%$ of clear cell and $20 \%$ of mucinous tumours.

Immunostaining with CD31 identified microvessels (Figure 1F). Median vascular was 18.44 (range 7.6-39.75). A total of $61 \%$ of tumours had vascular density counts below the median, high
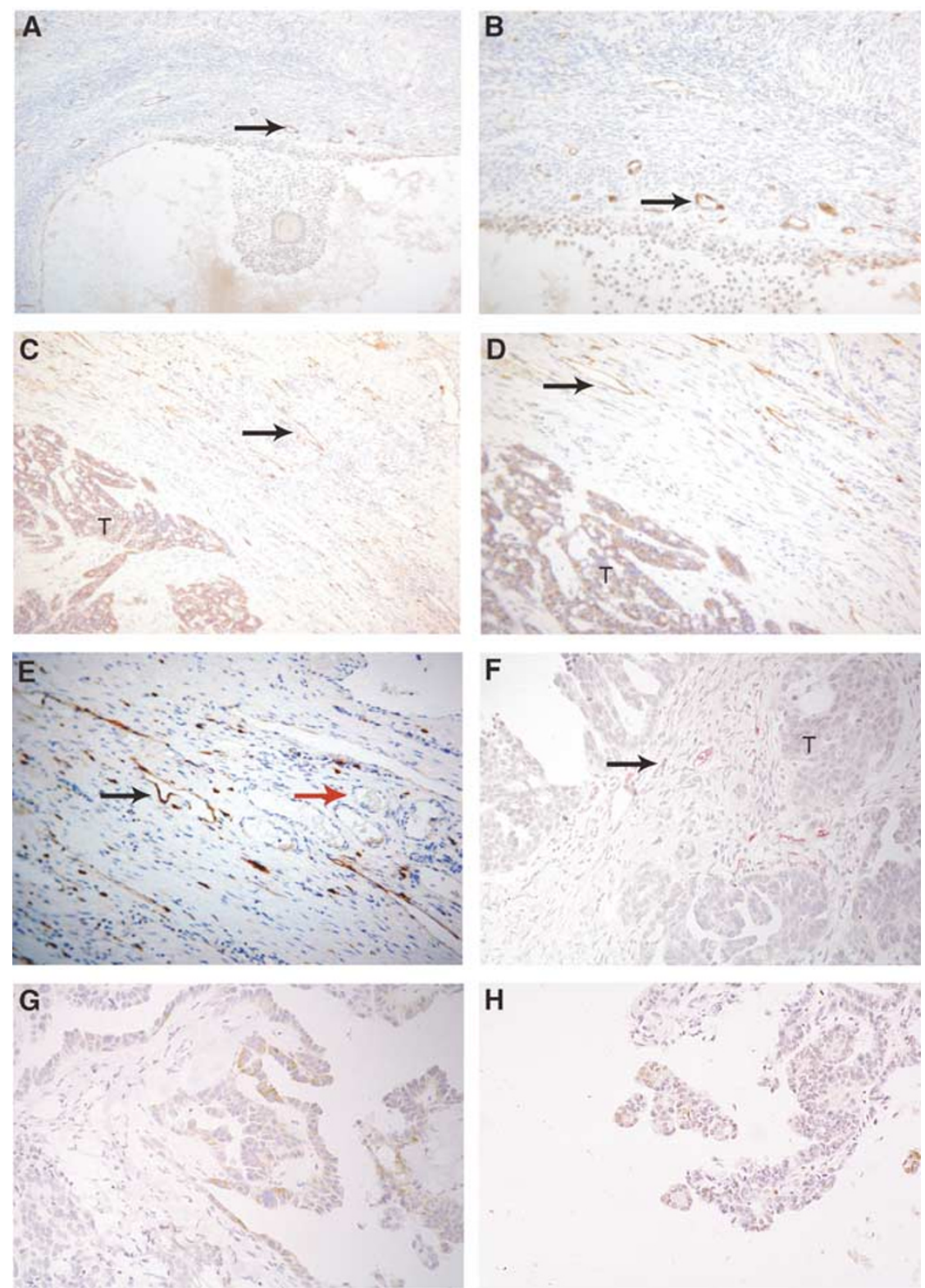

Figure I Immunostaining of normal ovary and epithelial ovarian cancer with antibodies. Perifollicular lymphatics in normal ovary at $\times 10$ and $\times 20$ magnification $(\mathbf{A}$ and $\mathbf{B})$. Lymphatic vessels adjacent to tumour at $\times 10, \times 20$ and $\times 40(\mathbf{C}-\mathbf{E})$. Blood vessels containing RBC are not stained with LYVE-I (marked with red arrow). Blood vessels in tumour at $\times 20(\mathbf{F})$. Vascular endothelial growth factor staining of tumour epithelium at $\times 20(V E G F)(\mathbf{G})$. Epithelial staining of tumour $($ TP) at $\times 20(\mathbf{H})$. Arrowheads identify vessels and T marks tumour. 
vascular counts $(>30)$ were noted in $0 \%$ of serous, $7 \%$ of endometroid, 7\% clear cell and 27\% mucinous tumours (Supplementary data Table 1).

Vascular endothelial growth factor staining was noted in various sites (stromal, macrophage, epithelial and vascular) and were quantified (Figure 1G) whereas TP staining (Figure 1H) was noted in the stroma and epithelium of the ovary. Strong staining of VEGF was noted in $29 \%$ (epithelial), $17 \%$ (stromal), 43\% (macrophage) and $19 \%$ (vascular) of tumours. Strong staining with TP was noted in $1 \%$ (epithelial) and $31 \%$ (stromal) of tumours (Supplementary data Table 1).

\section{Statistical analysis}

In univariate analysis (Table 2$)$, age $(P<0.001)$, residual disease $(P<0.001)$ and FIGO stage $(P<0.001)$ were associated with a significantly shorter overall survival and progression-free survival.

Table 2 Univariate analysis on the association of the variables with the outcome measures

\begin{tabular}{|c|c|c|c|c|c|c|}
\hline \multirow[b]{2}{*}{ Variable } & \multicolumn{3}{|c|}{ Progression-free survival } & \multicolumn{3}{|c|}{ Overall survival } \\
\hline & HR & $95 \% \mathrm{Cl}$ & $P$-value & HR & $95 \% \mathrm{Cl}$ & $P$-valu \\
\hline Age & 1.03 & $1.01-1.05$ & 0.01 & 1.03 & $1.01-1.05$ & \\
\hline \multicolumn{7}{|l|}{ Residual disease } \\
\hline Microscopic & Baseline & & & Baseline & & \\
\hline$\leqslant 2 \mathrm{~cm}$ & 2.50 & $1.42-4.40$ & 0.001 & 2.06 & $1.20-3.54$ & 0.0 \\
\hline$\geqslant 2 \mathrm{~cm}$ & 7.07 & $3.94-12.67$ & $>0.001$ & 7.94 & $4.48-14.08$ & $8<0.00$ \\
\hline \multicolumn{7}{|l|}{ Stage } \\
\hline 1 & Baseline & & & Baseline & & \\
\hline$\|$ & 2.14 & $0.92-4.95$ & 0.08 & 2.27 & $1.03-5.04$ & 0.0 \\
\hline III & 3.98 & $2.16-7.34$ & $<0.001$ & 3.78 & $2.12-6.74$ & $<0.00$ \\
\hline IV & 6.69 & $2.82-15.86$ & $<<0.001$ & 5.33 & $2.33-12.15$ & $5<0.00$ \\
\hline
\end{tabular}

Histology

Endometrioid

Clear cell

Mucinous

Serous

Others

Baseline

Baseline

\section{$0.63 \quad 0.31-1.34$}

$0.7|\quad 0.3|-1.65$

$0.88 \quad 0.51-1.53$

$1.25 \quad 0.54-2.89$

0.24
0.43

$0.65 \quad 0.31-1.34$

0.62
$0.65-1.50$

$\begin{array}{lll}0.65 & 1.13 & 0.68-1.89\end{array}$

$\begin{array}{lll}0.60 & 1.35 & 0.59-3.11\end{array}$

0.24

0.31

0.63

Differentiation

Well

Moderate

Moderate-poor

Baseline

$\begin{array}{ll}1.42 & 0.73-2.73\end{array}$

$0.77 \quad 0.62-5.04$

$1.92 \quad 0.95-3.87$

Baseline

Poor

$1.01 \quad 0.99-1.03$

$0.99 \quad 0.95-1.02$

$\begin{array}{lll}0.30 & 1.32 & 0.72-2.43 \\ 0.29 & 1.80 & 0.69-4.69\end{array}$

$\begin{array}{lll}0.29 & 1.80 & 0.69-4.69 \\ 0.07 & 1.59 & 0.81-3.12\end{array}$

0.37

Lymphatic count

Vascular count

$1.00 \quad 0.81-1.24$

$\begin{array}{lll}0.30 & 1.01 & 0.99-1.03\end{array}$

$\begin{array}{lll}0.53 & 0.98 & 0.95-1.02\end{array}$

0.34

TP

Stroma

$0 / I$ vs 2 vs 3

$0 / 1 / 2$ vs 3

$0 / I$ vs $2 / 3$

Epithelium

$\begin{array}{ll}1.01 & 0.76-1.35\end{array}$

$1.24 \quad 0.73-2.08$

$0.86 \quad 0.53-1.4 \mid$

$1.21 \quad 0.73-2.04$

\subsection{6}

$1.11 \quad 0.90-1.38$

$\begin{array}{lll}0.94 & 1.14 & 0.85-1.5 \\ 0.43 & 1.44 & 0.86-2.40\end{array}$

$\begin{array}{lll}0.43 & 1.44 & 0.86-2.40\end{array}$

$\begin{array}{lll}0.56 & 1.06 & 0.65-1.74\end{array}$

$\begin{array}{lll}0.46 & 1.07 & 0.63-1.80\end{array}$

0.33

0.38

0.17

0.81

VEGF

Epithelial

Macrophage

Stromal

Vascular

$0.97 \quad 0.79-1.81$

$1.01 \quad 0.84-1.23$

$1.01 \quad 0.80-1.28$

$0.98 \quad 0.80-1.20$

$\begin{array}{llll}0.74 & 0.97 & 0.80-1.19 & 0.79\end{array}$

$\begin{array}{llll}0.89 & 1.02 & 0.85-1.24 & 0.8\end{array}$

$\begin{array}{llll}0.94 & 0.99 & 0.78-1.26 & 0.94\end{array}$

$\begin{array}{llll}0.83 & 0.97 & 0.79-1.19 & 0.80\end{array}$

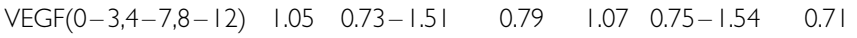

$\mathrm{Cl}=$ confidence intervals; $\mathrm{HR}=$ hazards ratios; $\mathrm{TP}=$ thymidine phosphorylase $\mathrm{VEGF}=$ vascular endothelial growth factor. Age was analyzed as a continuous variable. Univariate analysis of TP and VEGF expression was performed by considering the trend in expression.
In this set of patients, histological type or grade of differentiation did not show impact on progression-free or overall survival. Neither lymphatic nor vascular counts were statistically significant on univariate analysis. An analysis of VEGF staining in various sites and TP did not reveal any significance in univariate analysis.

On multivariate analysis (Table 3 ) two different mathematical models - one with residual disease and the other with stage were used to determine whether lymphatic density was an independent

Table 3 Multivariate analysis on the association of the variables with outcome measures. 3A Lymphatic count, 3B Vascular count, VEGF and TP as variables

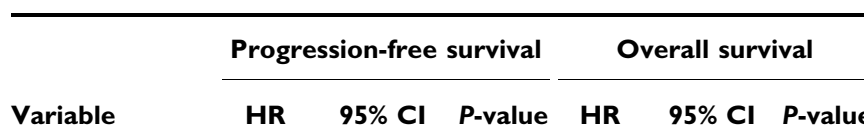

\section{(A)}

Model 1

$\begin{array}{lllllll}\text { Age } & 1.02 & 1.01-1.05 & 0.07 & 1.03 & 1.01-1.05 & 0.01\end{array}$

$\begin{array}{lllllll}\text { Lymphatic count } & 1.02 & 1.00-1.05 & 0.05 & 1.02 & 1.00-1.04 & 0.04\end{array}$

Residual disease

\begin{tabular}{lcccccc} 
Microscopic & Baseline & \multicolumn{5}{c}{ Baseline } \\
$\leqslant 2 \mathrm{~cm}$ & 2.21 & $1.16-4.20$ & 0.02 & 1.64 & $0.088-3.06$ & 0.12 \\
$\geqslant 2 \mathrm{~cm}$ & 7.38 & $3.77-14.46$ & $<0.001$ & 7.43 & $3.86-|4.3|$ & $<0.00 \mid$
\end{tabular}

Model II

$\begin{array}{lllllll}\text { Age } & 1.02 & 1.00-1.05 & 0.05 & 1.03 & 1.00-1.05 & 0.02 \\ \text { Lymphatic count } & 1.01 & 0.99-1.03 & 0.27 & 1.01 & 0.99-1.03 & 0.19\end{array}$

Stage

II
II
IV

\begin{tabular}{cccrrr} 
Baseline & \multicolumn{5}{c}{ Baseline } \\
1.57 & $0.63-3.94$ & 0.34 & 1.62 & $0.68-3.84$ & 0.27 \\
3.31 & $1.74-6.31$ & $<0.001$ & 3.05 & $1.66-5.60$ & $<0.001$ \\
6.26 & $2.63-14.90$ & $<0.001$ & 4.21 & $1.88-9.68$ & $<0.001$
\end{tabular}

(B)

Model I

Age

$1.02 \quad 0.99-1.04$

$\begin{array}{lll}0.11 & 1.03 & 1.01-1.05\end{array}$

0.04
Residual disease
Microscopic
$\leqslant 2 \mathrm{~cm}$
$\geqslant 2 \mathrm{~cm}$
Vascular count
VEGFe
VEGFm
VEGFs
VEGFV
TPS
$\mathrm{TPe}$

\begin{tabular}{cccccc} 
Baseline & \multicolumn{5}{c}{ Baseline } \\
1.93 & $1.104-3.58$ & 0.04 & 1.79 & $0.97-3.22$ & 0.06 \\
6.77 & $3.58-12.81$ & $<0.001$ & 7.63 & $4.00-14.58$ & $<0.001$ \\
& & & & & \\
0.82 & $0.48-1.40$ & 0.46 & 0.77 & $0.44-1.34$ & 0.35 \\
1.02 & $0.83-1.25$ & 0.85 & 1.03 & $0.84-1.28$ & 0.75 \\
0.99 & $0.82-1.20$ & 0.93 & 0.98 & $0.81-1.19$ & 0.85 \\
1.10 & $0.86-1.41$ & 0.45 & 1.07 & $0.83-1.38$ & 0.59 \\
1.14 & $0.92-1.42$ & 0.24 & 1.13 & $0.91-1.41$ & 0.26 \\
0.97 & $0.79-1.20$ & 0.81 & 1.07 & $0.86-1.32$ & 0.55 \\
1.36 & $0.79-2.33$ & 0.27 & 1.09 & $0.63-1.88$ & 0.76
\end{tabular}

Model II

Age

$1.01 \quad 0.99-1.04$

0.34

$1.020 .99-1.04$

0.16

Stage
I
II
III
IV

\begin{tabular}{cccccl} 
Baseline & \multicolumn{5}{c}{ Baseline } \\
2.07 & $0.85-5.00$ & 0.11 & 2.25 & $0.95-5.32$ & 0.07 \\
3.41 & $1.77-6.59$ & $<0.001$ & 3.11 & $1.61-6.04$ & 0.001 \\
4.44 & $1.63-12.10$ & $<0.001$ & 4.79 & $1.74-13.18$ & 0.002 \\
& & & & & \\
0.97 & $0.57-1.65$ & 0.90 & 0.95 & $0.56-1.62$ & 0.85 \\
1.04 & $0.94-1.29$ & 0.73 & 1.05 & $0.84-1.30$ & 0.68 \\
1.01 & $0.83-1.23$ & 0.90 & 1.00 & $0.82-1.22$ & 1.00 \\
1.11 & $0.87-1.41$ & 0.40 & 1.06 & $0.83-1.35$ & 1.00 \\
1.11 & $0.89-1.39$ & 0.33 & 1.11 & $0.89-1.38$ & 0.37 \\
1.02 & $0.82-1.26$ & 0.89 & 1.12 & $0.90-1.40$ & 0.29 \\
1.28 & $0.74-2.19$ & 0.38 & 0.99 & $0.57-1.70$ & 0.96 \\
\hline
\end{tabular}

$\mathrm{Cl}=$ confidence intervals; $\mathrm{HR}=$ hazards ratios; $\mathrm{TP}=$ thymidine phosphorylase: VEGF $=$ vascular endothelial growth factor. $T$ 
prognostic factor as residual disease and stage are closely related. When all of the significant prognostic factors were taken into account simultaneously in a Cox proportional hazards model, the amount of residual disease was the strongest independent prognostic indicator for overall survival and progression-free survival $(P<0.001)$. An analysis of this data set using the Cox proportional hazards model comprising residual disease revealed that lymphatic density reached statistical significance in progression-free survival $(P=0.05$, hazards ratio $(\mathrm{HR}) 1.00-1.05)$ and overall survival $(P=0.04, \mathrm{HR} 1.00-1.04)$. When this analysis was repeated with another mathematical model comprising stage and age, LVD was not shown to be of statistical significance. Multivariate analysis using a model with residual disease indicated that the vascular count was not associated with either overall or progression-free survival. Mann-Whitney test did not reveal any statistical significance for either $\operatorname{LVD}(P=0.10)$ or MVD $(P=0.87)$ for response to treatment. Similarly, expression levels of VEGF and TP were not statistically significant in either overall survival or progression-free survival using either of the two models.

A Kaplan-Meier curve of survival (Figure 2) using a median value of 18 lymphatic vessels as a cutoff did not show any discrimination in survival with high- or low-lymphatic counts. In addition, lymphatic count did not show any correlation with residual disease (Krusker-Wallis $P=0.53$, analysis of variance $P=0.44$ ) or with histology, differentiation of tumour or vascular count. Lymphatic count did not predict any difference in survival curves for the whole series or in a subset of Stage I patients.
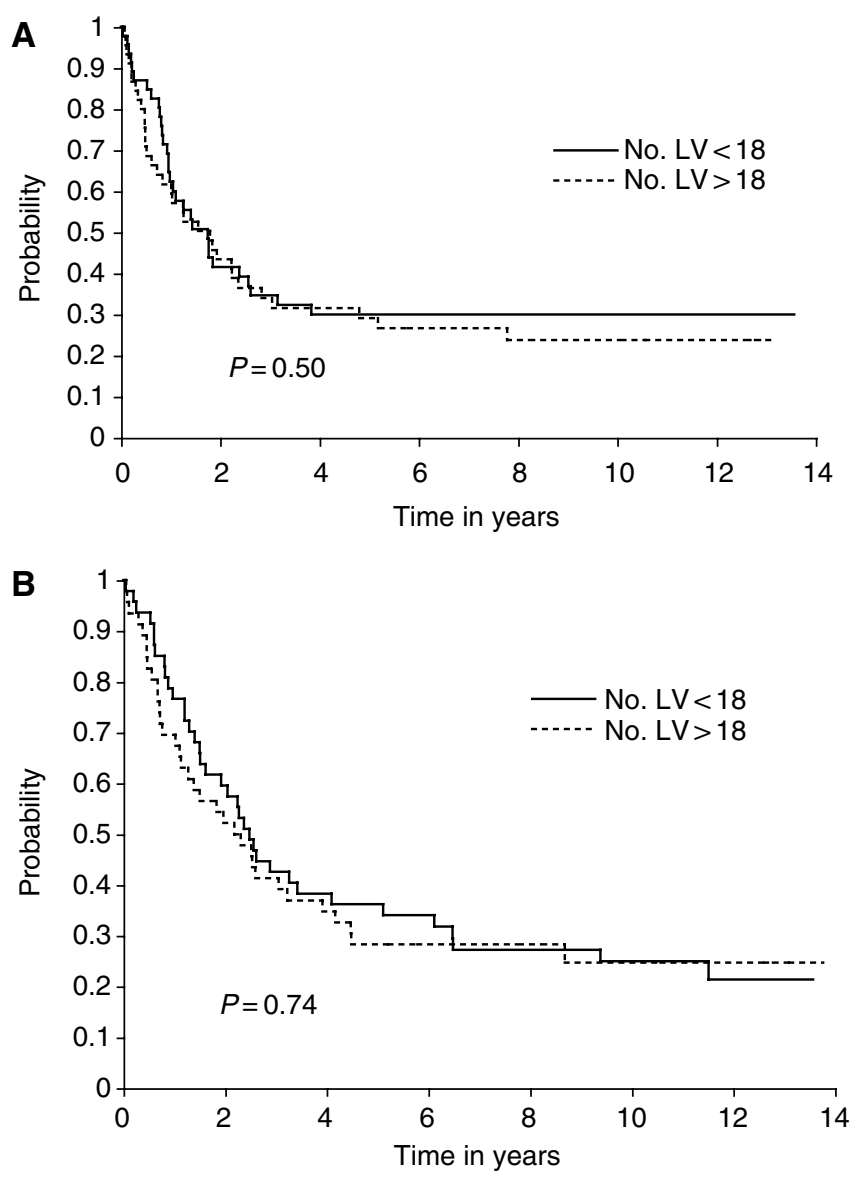

Figure 2 Kaplan-Meier survival plots of lymphatic vessel density in ovarian cancer as an outcome measure for progression-free $(\mathbf{A})$ and overall survival (B). Lymphatic vessel counts are denoted as $<$ or $>18$ as indicated. Number of patients $=93$

\section{DISCUSSION}

The recent identification of immunohistochemical markers that can reliably distinguish lymphatic vessels from blood vessels in tissue sections has yielded new insight into the mechanisms of metastasis. We investigated LVD, vascular density and angiogenic factors in epithelial ovarian cancer in order to understand the relative contributions of lymphatic and vascular spread in ovarian cancer. This is the first report of LVD in ovarian cancer as measured by LYVE-1, one of the best-characterised markers for lymph vessels.

Our study found that on univariate analysis, both progression-free and overall survival decreased significantly with age, in patients with FIGO stage greater than II and in patients with residual disease after surgery. This is consistent with published literature and clinical practice. In multivariate analysis, using a model adjusting for age and residual disease, LVD was statistically significant in progressionfree and overall survival. This was not replicated in another model comprising age and stage of disease. A previous study in epithelial ovarian cancer using a polyclonal antibody to podoplanin (Birner et al, 2000) showed that intratumoural LVD was not a statistically significant variable in overall or progression-free survival. This study differed from the previous study in the following ways. We used LYVE-1 as a lymphatic marker. It has been shown previously that these antibodies/markers delineate identical lymphatic vessels on tissue sections (Straume and Akslen, 2004). Secondly, we counted three hotspots as opposed to one hotspot, which may give a more representative view of the number of lymphatic vessels in the tumour. We did not discriminate between intra- and peri-tumoral vessels as ovarian cancer frequently penetrates the ovarian capsule and assessment of LVD as a whole may be relevant in ovarian cancer.

Lymphovascular invasion in tissue sections of tumours has been clearly documented to be a reliable prognostic variable predicting nodal metastasis and survival in breast, cervix cancer and impacts on decision making for therapy in testicular cancer (Warde et al, 1997; Morice et al, 2003; Schoppmann et al, 2004; Dinshaw et al, 2005). However, the prognostic significance of LVD in cancer is not clearcut. In head and neck cancer, studies show an association of increased LVD and new vessel formation with lymph node metastasis and worse prognosis (Beasley et al, 2002; Maula et al, 2003). In melanoma, one large study with 202 samples demonstrated improved overall and recurrence free survival with increased LVD in both peritumoural and intratumoural areas (Straume et al, 2003) suggesting that vessel density may be a marker for an improved immune response whereas two smaller studies conclude the opposite with intratumoural lymphatics significantly higher in metastatic melanomas and correlating with poor survival (Dadras et al, 2003; Shields et al, 2004). Interestingly, in breast cancer intratumoral lymphatic vessels are absent and the significance of peritumoural vessels is unclear (Williams et al, 2003; Bono et al, 2004; Vleugel et al, 2004). Further studies in other tumour types investigating LVD and proliferation markers may clarify the role of new lymph vessel formation and the significance of LVD in cancer progression.

Published studies with blood vessel density in ovarian cancers are equivocal with some studies revealing an association with poor prognosis whereas others dispute this (Hollingsworth et al, 1995; Orre et al, 1998; Alvarez et al, 1999; Abulafia et al, 2000; Nakayama et al, 2001). We found that MVD in an overlapping set of tumours did not influence prognosis or survival or response to treatment. Both VEGF and TP are important angiogenic factors for many malignancies and have been postulated to work synergistically (Moghaddam et al, 1995; Ferrara, 2004). An analysis of the expression of these angiogenic factors in our set of tumours did not reveal any impact on survival. This is similar to some published studies but is in contrast to others (Moghaddam et al, 1995; Nishida et al, 2004; Raspollini et al, 2004; Sonmezer et al, 2004). 
Lymphatic vessel density in our series was statistically significant in multivariate analysis in overall survival and progression-free survival. Lymphatic counts did not however, discriminate in survival curves. This would suggest that lymphatic spread does not have absolute prognostic significance but may act in conjunction with other biological factors to aid metastasis in ovarian cancer. A larger study of Stage I ovarian cancers will be useful in establishing the prognostic value of LVD and whether this can be used to predict the need for chemotherapy in these patients. Ovarian cancer is primarily an abdominal disease and our study would support the view that intraperitoneal spread of ovarian cancer occurs through direct dissemination of disease rather than through lymphatic or vascular spread.

\section{REFERENCES}

Abulafia O, Ruiz JE, Holcomb K, Dimaio TM, Lee YC, Sherer DM (2000) Angiogenesis in early-invasive and low-malignant-potential epithelial ovarian carcinoma. Obstet Gynecol 95: 548-552

Agarwal R, Kaye SB (2003) Ovarian cancer: strategies for overcoming resistance to chemotherapy. Nat Rev Cancer 3: 502-516

Alvarez AA, Krigman HR, Whitaker RS, Dodge RK, Rodriguez GC (1999) The prognostic significance of angiogenesis in epithelial ovarian carcinoma. Clin Cancer Res 5: 587 - 591

Bamberger ES, Perrett CW (2002) Angiogenesis in epithelian ovarian cancer. Mol Pathol 55: 348-359

Banerji S, Ni J, Wang SX, Clasper S, Su J, Tammi R, Jones M, Jackson DG (1999) LYVE-1, a new homologue of the CD44 glycoprotein, is a lymphspecific receptor for hyaluronan. J Cell Biol 144: 789-801

Beasley NJ, Prevo R, Banerji S, Leek RD, Moore J, van Trappen P, Cox G, Harris AL, Jackson DG (2002) Intratumoral lymphangiogenesis and lymph node metastasis in head and neck cancer. Cancer Res 62: $1315-1320$

Birner P, Schindl M, Obermair A, Plank C, Breitenecker G, Kowalski H, Oberhuber G (2000) Lymphatic microvessel density in epithelial ovarian cancer: its impact on prognosis. Anticancer Res 20: $2981-2985$

Bono P, Wasenius VM, Heikkila P, Lundin J, Jackson DG, Joensuu H (2004) High LYVE-1-positive lymphatic vessel numbers are associated with poor outcome in breast cancer. Clin Cancer Res 10: 7144-7149

Breiteneder-Geleff S, Soleiman A, Kowalski H, Horvat R, Amann G, Kriehuber E, Diem K, Weninger W, Tschachler E, Alitalo K, Kerjaschki D (1999) Angiosarcomas express mixed endothelial phenotypes of blood and lymphatic capillaries: podoplanin as a specific marker for lymphatic endothelium. Am J Pathol 154: 385-394

Cannistra SA (2004) Cancer of the ovary. N Engl J Med 351: 2519-2529

Cox DR, Oakes D (1984) Analysis of Survival Data. London: Chapman \& Hall

Dadras SS, Paul T, Bertoncini J, Brown LF, Muzikansky A, Jackson DG, Ellwanger U, Garbe C, Mihm MC, Detmar M (2003) Tumor lymphangiogenesis: a novel prognostic indicator for cutaneous melanoma metastasis and survival. Am J Pathol 162: $1951-1960$

Dinshaw KA, Budrukkar AN, Chinoy RF, Sarin R, Badwe R, Hawaldar R, Shrivastava SK (2005) Profile of prognostic factors in 1022 Indian women with early-stage breast cancer treated with breast-conserving therapy. Int J Radiat Oncol Biol Phys 63(4): 1132-1141

Ferrara N (2004) Vascular endothelial growth factor: basic science and clinical progress. Endocr Rev 25: 581 -611

Ferrara N, Alitalo K (1999) Clinical applications of angiogenic growth factors and their inhibitors. Nat Med 5: 1359-1364

Fox SB, Harris AL (2004) Histological quantitation of tumour angiogenesis. Apmis 112: $413-430$

Fox SB, Moghaddam A, Westwood M, Turley H, Bicknell R, Gatter KC, Harris AL (1995) Platelet-derived endothelial cell growth factor/ thymidine phosphorylase expression in normal tissues: an immunohistochemical study. J Pathol 176: $183-190$

Hollingsworth HC, Kohn EC, Steinberg SM, Rothenberg ML, Merino MJ (1995) Tumor angiogenesis in advanced stage ovarian carcinoma. Am J Pathol 147: $33-41$

Horak ER, Leek R, Klenk N, LeJeune S, Smith K, Stuart N, Greenall M, Stepniewska K, Harris AL (1992) Angiogenesis, assessed by platelet/ endothelial cell adhesion molecule antibodies, as indicator of node metastases and survival in breast cancer. Lancet 340: 1120-1124

\section{ACKNOWLEDGEMENTS}

We acknowledge the Immunohistochemistry services provided by the Department of Histopathology at UCL, London. SS was funded by a research training fellowship from Oxfordshire Health Services Research Committee (OHSRC), Cancer Research UK and from the Department of Gynaecological Oncology, Cheltenham General Hospital, Cheltenham.

Supplementary Information accompanies the paper on British Journal of Cancer website (http://www.nature.com/bjc)
Jackson DG, Prevo R, Clasper S, Banerji S (2001) LYVE-1, the lymphatic system and tumor lymphangiogenesis. Trends Immunol 22: 317-321

Kabbinavar FF, Hambleton J, Mass RD, Hurwitz HI, Bergsland E, Sarkar S (2005) Combined analysis of efficacy: the addition of bevacizumab to fluorouracil/leucovorin improves survival for patients with metastatic colorectal cancer. J Clin Oncol 23: 3706-3712

Kaipainen A, Korhonen J, Mustonen T, van Hinsbergh VW, Fang GH, Dumont D, Breitman M, Alitalo K (1995) Expression of the fms-like tyrosine kinase 4 gene becomes restricted to lymphatic endothelium during development. Proc Natl Acad Sci USA 92: $3566-3570$

Kaplan EL, Meier P (1958) Nonparametric Estimation from incomplete observations. J Am Stat Assoc 53: $457-481$

Knudson CB, Knudson W (1993) Hyaluronan-binding proteins in development, tissue homeostasis, and disease. FASEB J 7: 1233-1241

Knudson W, Bartnik E, Knudson CB (1993) Assembly of pericellular matrices by COS-7 cells transfected with CD44 lymphocyte-homing receptor genes. Proc Natl Acad Sci USA 90: 4003-4007

Makinen T, Jussila L, Veikkola T, Karpanen T, Kettunen MI, Pulkkanen KJ, Kauppinen R, Jackson DG, Kubo H, Nishikawa S, Yla-Herttuala S, Alitalo K (2001) Inhibition of lymphangiogenesis with resulting lymphedema in transgenic mice expressing soluble VEGF receptor-3. Nat Med 7: 199-205

Mandriota SJ, Jussila L, Jeltsch M, Compagni A, Baetens D, Prevo R, Banerji S, Huarte J, Montesano R, Jackson DG, Orci L, Alitalo K, Christofori G, Pepper MS (2001) Vascular endothelial growth factor-Cmediated lymphangiogenesis promotes tumour metastasis. EMBO J 20: $672-682$

Maula SM, Luukkaa M, Grenman R, Jackson D, Jalkanen S, Ristamaki R (2003) Intratumoral lymphatics are essential for the metastatic spread and prognosis in squamous cell carcinomas of the head and neck region. Cancer Res 63: 1920-1926

Moghaddam A, Zhang HT, Fan TP, Hu DE, Lees VC, Turley H, Fox SB, Gatter KC, Harris AL, Bicknell R (1995) Thymidine phosphorylase is angiogenic and promotes tumor growth. Proc Natl Acad Sci USA 92: 998- 1002

Morice P, Piovesan P, Rey A, Atallah D, Haie-Meder C, Pautier P, Sideris L, Pomel C, Duvillard P, Castaigne D (2003) Prognostic value of lymphovascular space invasion determined with hematoxylin-eosin staining in early stage cervical carcinoma: results of a multivariate analysis. Ann Oncol 14: $1511-1517$

Mouta Carreira C, Nasser SM, di Tomaso E, Padera TP, Boucher Y, Tomarev SI, Jain RK (2001) LYVE-1 is not restricted to the lymph vessels: expression in normal liver blood sinusoids and down-regulation in human liver cancer and cirrhosis. Cancer Res 61: $8079-8084$

Nakayama K, Kanzaki A, Takebayashi Y, Toi M, Bando H, Nabei T, Miyazaki K, Fukumoto M (2001) Different features of angiogenesis between ovarian and breast carcinoma. Cancer Lett 170: 161-167

Nishida N, Yano H, Komai K, Nishida T, Kamura T, Kojiro M (2004) Vascular endothelial growth factor $\mathrm{C}$ and vascular endothelial growth factor receptor 2 are related closely to the prognosis of patients with ovarian carcinoma. Cancer 101: $1364-1374$

Oh SJ, Jeltsch MM, Birkenhager R, McCarthy JE, Weich HA, Christ B, Alitalo K, Wilting J (1997) VEGF and VEGF-C: specific induction of angiogenesis and lymphangiogenesis in the differentiated avian chorioallantoic membrane. Dev Biol 188: 96-109 
Orre M, Lotfi-Miri M, Mamers P, Rogers PA (1998) Increased microvessel density in mucinous compared with malignant serous and benign tumours of the ovary. Br J Cancer 77: 2204-2209

Pepper MS (2001) Lymphangiogenesis and tumor metastasis: myth or reality? Clin Cancer Res 7: $462-468$

Prevo R, Banerji S, Ferguson DJ, Clasper S, Jackson DG (2001) Mouse LYVE-1 is an endocytic receptor for hyaluronan in lymphatic endothelium. J Biol Chem 276: 19420 - 19430

Raspollini MR, Amunni G, Villanucci A, Baroni G, Boddi V, Taddei GL (2004) Prognostic significance of microvessel density and vascular endothelial growth factor expression in advanced ovarian serous carcinoma. Int I Gynecol Cancer 14: 815-823

Scavelli C, Weber E, Agliano M, Cirulli T, Nico B, Vacca A, Ribatti D (2004) Lymphatics at the crossroads of angiogenesis and lymphangiogenesis. J Anat 204: 433 - 449

Schoppmann SF, Bayer G, Aumayr K, Taucher S, Geleff S, Rudas M, Kubista E, Hausmaninger H, Samonigg H, Gnant M, Jakesz R, Horvat R (2004) Prognostic value of lymphangiogenesis and lymphovascular invasion in invasive breast cancer. Ann Surg 240: 306-312

Shields JD, Borsetti M, Rigby H, Harper SJ, Mortimer PS, Levick JR, Orlando A, Bates DO (2004) Lymphatic density and metastatic spread in human malignant melanoma. Br J Cancer 90: 693-700

Shimizu K, Kubo H, Yamaguchi K, Kawashima K, Ueda Y, Matsuo K, Awane M, Shimahara Y, Takabayashi A, Yamaoka Y, Satoh S (2004) Suppression of VEGFR-3 signaling inhibits lymph node metastasis in gastric cancer. Cancer Sci 95: $328-333$

Skobe M, Hawighorst T, Jackson DG, Prevo R, Janes L, Velasco P, Riccardi L, Alitalo K, Claffey K, Detmar M (2001) Induction of tumor lymphangiogenesis by VEGF-C promotes breast cancer metastasis. Nat Med 7: $192-198$

Sonmezer M, Gungor M, Ensari A, Ortac F (2004) Prognostic significance of tumor angiogenesis in epithelial ovarian cancer: in association with transforming growth factor beta and vascular endothelial growth factor. Int J Gynecol Cancer 14: $82-88$

Stacker SA, Caesar C, Baldwin ME, Thornton GE, Williams RA, Prevo R, Jackson DG, Nishikawa S, Kubo H, Achen MG (2001) VEGF-D promotes the metastatic spread of tumor cells via the lymphatics. Nat Med 7: $186-191$
Straume O, Akslen LA (2004) Lymphatic vessel density and prognosis in cutaneous melanoma. Br J Cancer 91: 1224-1225

Straume O, Jackson DG, Akslen LA (2003) Independent prognostic impact of lymphatic vessel density and presence of low-grade lymphangiogenesis in cutaneous melanoma. Clin Cancer Res 9: 250-256

Toi M, Atiqur Rahman M, Bando H, Chow LW (2005) Thymidine phosphorylase (platelet-derived endothelial-cell growth factor) in cancer biology and treatment. Lancet Oncol 6: 158-166

Turley H, Scott PA, Watts VM, Bicknell R, Harris AL, Gatter KC (1998) Expression of VEGF in routinely fixed material using a new monoclonal antibody VG1. J Pathol 186: $313-318$

Uzzan B, Nicolas P, Cucherat M, Perret GY (2004) Microvessel density as a prognostic factor in women with breast cancer: a systematic review of the literature and meta-analysis. Cancer Res 64: 2941 - 2955

Van der Auwera I, Van Laere SJ, Van den Eynden GG, Benoy I, van Dam P, Colpaert CG, Fox SB, Turley H, Harris AL, Van Marck EA, Vermeulen PB, Dirix LY (2004) Increased angiogenesis and lymphangiogenesis in inflammatory versus noninflammatory breast cancer by real-time reverse transcriptase-PCR gene expression quantification. Clin Cancer Res 10: $7965-7971$

Vermeulen PB, Gasparini G, Fox SB, Colpaert C, Marson LP, Gion M, Belien JA, de Waal RM, Van Marck E, Magnani E, Weidner N, Harris AL, Dirix LY (2002) Second international consensus on the methodology and criteria of evaluation of angiogenesis quantification in solid human tumours. Eur J Cancer 38: $1564-1579$

Vleugel MM, Bos R, van der Groep P, Greijer AE, Shvarts A, Stel HV, van der Wall E, van Diest PJ (2004) Lack of lymphangiogenesis during breast carcinogenesis. J Clin Pathol 57: 746 - 751

Warde P, Gospodarowicz MK, Banerjee D, Panzarella T, Sugar L, Catton CN, Sturgeon JF, Moore M, Jewett MA (1997) Prognostic factors for relapse in stage I testicular seminoma treated with surveillance. J Urol 157: $1705-1709$

Wigle JT, Harvey N, Detmar M, Lagutina I, Grosveld G, Gunn MD, Jackson DG, Oliver G (2002) An essential role for Prox1 in the induction of the lymphatic endothelial cell phenotype. EMBO J 21: 1505-1513

Williams CS, Leek RD, Robson AM, Banerji S, Prevo R, Harris AL, Jackson DG (2003) Absence of lymphangiogenesis and intratumoural lymph vessels in human metastatic breast cancer. J Pathol 200: 195-206 